\title{
The Middle-East-respiratory-syndrome coronavirus: the management
}

\begin{abstract}
The Middle-East-Respiratory-Syndrome coronavirus (MERS-CoV) is a beta coronavirus. Camel is the primary source of MERS-CoV. The incubation period is up to 14 days. The Kingdom of Saudi Arabia is the major source of reported cases. Case definition of MERS$\mathrm{CoV}$ can be classified to person under investigation (PUI), probable case, presumptive confirmed case, and confirmed case. Transmission of MERS-CoV is mainly via droplets. Currently, there is no specific antiviral therapy for MERS-CoV infection, only infection precaution is effective. Larger clinical trials are needed to identify the effective antiviral therapy.
\end{abstract}

Keywords: MERS-CoV, Management, Epidemiology, Diagnosis, MERS-CoV, Infected, Antiviral therapy, Clinical trials
Volume 2 Issue 6 - 2015

\author{
Attapon Cheepsattayakorn, ${ }^{1,2,3}$ Ruangrong \\ Cheepsattayakorn ${ }^{4}$ \\ 'Senior Editor, Journal of Human Virology and Retrovirology, \\ USA \\ ${ }^{2}$ IOth Zonal Tuberculosis and Chest Disease Center, Thailand \\ ${ }^{3}$ IOth Office of Disease Prevention and Control, Department of \\ Disease Control, Ministry of Public Health, Thailand \\ ${ }^{4}$ Department of Pathology, Faculty of Medicine, Chiang Mai \\ University, Thailand
}

Correspondence: Attapon Cheepsattayakorn, I0th Zonal Tuberculosis and Chest Disease Center, 143 Sridornchai Road Changklan Muang Chiang Mai 50100,Thailand, Tel 6653 I40767, 6653 276364, Fax 6653 140773, 6653273590 ,

Email attapon1958@gmail.com, attaponche@yahoo.com

Received: October 16, 2015 | Published: October 29, 2015
Abbreviations: KSA, Kingdom of Saudi Arabia; WHO, World Health Organization; PUI, Person Under Investigation; PCR, Polymerase Chain Reaction; BAL, Bronchoalveolar Lavage; MERSCoV, Middle-East-Respiratory-Syndrome Coronavirus

\section{Introduction and epidemiological update}

Three major subgroups of coronaviruses are identified: alpha, beta, and gamma. ${ }^{1}$ The Middle-East-Respiratory-Syndrome Coronavirus (MERS-CoV), a beta coronavirus, has not been found in humans before. Individuals may be infected by close contact to animals, such as camel, confirmed cases or environment. Since April 2014, many secondary cases who were mainly healthcare workers appeared to be infected from patients suffering from MERS. Recent studies demonstrated that camel is the primary source of MERS-CoV.

The incubation period is up to 14 days. Fever, cough, and breathing difficulties are the main symptoms and most of the patients develop pneumonia. The patients may have atypically clinical presentation, such as diarrhea or renal failure. ${ }^{1}$ As of September 9, 2015, 1,542 cases of MERS-CoV have been reported to the World Health Organization (WHO) with at least 544 related deaths. ${ }^{2}$ Majority of them were reported from the Kingdom of Saudi Arabia (KSA) (89\%). Since 2012, at least 454 deaths were reported from KSA. ${ }^{3}$

Dromedary camels are likely to be the source of primary infection, but currently are due to human-to-human transmission. ${ }^{2}$ Sporadic zoonotic infections are its epidemiologic feature. Currently, evidence of sustained community transmission is not identified, both in the Middle East and South Korea. In KSA, during April-May 2014, occasional peaks of MERS-CoV cases appeared.

A second peak of cases occurred during February-May 2015, but the actual numbers of reported cases were significantly lower than the 2014 peak. Recently, an outbreak in Riyadh since July 31, 2015, is mainly related to outbreak in the King Abdul Aziz Medical Center-National Guard Hospital with 166 Riyadh reported cases, as of September 15, 2015. There were additionally possible-community cases in Riyadh though previous Nosocomial-exposure patterns. This could highlight MERS-CoV transmission within the KSA.

Additionally, local onward transmission in Jordan has been associated with a recently imported case to Jordan from the KSA, which is another indicator of the overall risk within the KSA. Hence, in the light of the upcoming Hajj and Umrah pilgrimage, careful observation is needed. In South Korea, 186 cases and 36 deaths were reported between May 20 and July 4, 2015 and no further cases have been declared since then. Finally, the WHO confirmed that the South Korean outbreak has ended. This South Korean outbreak indicated the importance of prompt and strict application of infection control measures. $^{2}$

\section{Virology of MERS-CoV}

MERS-CoV is a lineage $\mathrm{C}$ beta-coronavirus that closely related to several bat coronaviruses ${ }^{4-10}$ Dipeptidyl peptidase 4 (DPP4 or CD26), a functional receptor for MERS-CoV,,$^{11,12}$ displays high amino acid sequence conservation across different species, including the sequence obtained from bat cells. ${ }^{13}$ In the studies of cell-line susceptibility, human bronchial-epithelial cells were susceptible to MERS-CoV infection, ${ }^{14}$ but other several human cell lines can be infected, such as intestinal, hepatic, and renal cell Ines, including histiocytes. ${ }^{15}$ Additionally, MERS-CoV can infect nonhuman primate, bat, horse, rabbit, civet, and porcine cell lines. ${ }^{15-17}$ In a study in Saudi Arabia between June 2012 and June 2013, it revealed sufficient heterogeneity to support multiple separate animal-to-human transmissions. ${ }^{18}$ Viral isolates from Riyadh, Saudi Arabia, during the spring 2014, indicated that infections resulted from human-to-human transmission or increased zoonotic activity. ${ }^{13}$

\section{Case definition of MERS-CoV}

The Public Health Agency of Canada defines the case as the following: ${ }^{19}$ 
1. Person Under Investigation (PUI) is a person with acute respiratory illness that may include history of fever and new onset of cough (or exacerbation of chronic cough) or breathing difficulties with or without pulmonary parenchymal disease based on clinical or radiological evidence of consolidation and any of the following:

a. The person has history of travel to, or residence in, affected countries in the Middle East within 14 days before onset of illness.

b. The person has had close contact within 14 days before onset of illness with a person with acute respiratory illness of any degree who had history of travel to, or residence in affected countries in the Middle East within 14 days before the contact's illness.

c. The disease occurs as part of a cluster that occurs within a 14day period, without regard of to place of residence or history of travel, unless another etiology has been identified.

d. The disease occurs in a healthcare worker who has been working in an environment where patients with severe acute respiratory illness are being cared for, especially, patients requiring intensive care, without regard of to place of residence or history of travel, unless another etiology has been identified.

e. The person develops an unexpectedly severe clinical course despite appropriate treatment, even if another etiology has been identified, if that alternative does not fully explain the presentation or clinical course of the patient, or

f. A person with an acute respiratory illness of any degree of severity who, within 14 days before onset of illness, had close contact with a confirmed case, presumptive confirmed case, or probable case of MERS-CoV infection while the case was sick.

2. Probable Case is a person with an acute respiratory illness of any degree of severity who had close contact with a confirmed case or presumptive confirmed case and from whom laboratory diagnosis of MERS-CoV is unavailable or inconclusive.

A. Presumptive Confirmed Case is a person with a positive laboratory result of infection for MERS-CoV virus that is awaiting confirmation by the National Microbiology Laboratory (Canada).

B. Confirmed Case is a person with laboratory confirmation of infection with the MERS-CoV virus.

\section{Clinical manifestations and diagnosis}

The main clinical symptoms include fever, cough, breathlessness, and myalgia. ${ }^{20}$ Gastrointestinal symptoms may be present sometimes. ${ }^{20}$ Nasopharyngeal and or pharyngeal swabs must be taken together for polymerase chain reaction (PCR) testing. ${ }^{21}$ The sputum, bronchoalveolar lavage (BAL), end tracheal aspirate, and pleural fluid should be collected whenever clinically appropriate. ${ }^{21-25}$ For serum antibody testing, serum samples should be collected during the first week after onset of illness, and again, at least 3 weeks after the acute sample was collected (convalescent period). ${ }^{21}$ Nevertheless, single serum specimen collected 14 or more days after the clinical onset may be beneficial. For reverse transcriptase PCR (rRT-PCR) testing for detection of the antigen or virus, a single serum sample should be collected during the first week, particularly, 3-4 days after clinical onset. A second serum sample should be collected for antibody detection at least 14 days after the onset of illness. ${ }^{25}$

\section{Management of MERS-CoV infection}

The United States Centers for Disease Control and Prevention advices that: ${ }^{26}$

1) The people wash their hand with soap and water for 20 seconds. If soap and water are not available, use an alcohol-based hand sanitizer.

2) Covering their nose and mouth with a tissue when they cough or sneeze, then throw the tissue in the trash.

3) Avoiding touching their eyes, nose, and mouth with unwashed hands.

4) Avoiding personal contact with sick people.

5) Cleaning and disinfecting frequently touched surfaces and objects.

All patients who meet the case definition of suspected or contaminated MERS-CoV should be managed in a single room under transmission-based precautions, particularly, contact and airborne. ${ }^{27}$ The following patient placement options are advised according to facility resources. Option one: Single room with ensuite facilities, negative pressure air handling and dedicated anteroom. Option two: Single room with ensuite facilities without negative pressure air handling. Option three: Single room without ensuite facilities and without negative pressure. When the patients are cohorted, spatial separation of at least one meter should be maintained between each patient with curtain using for reduction of infection spreading. Donning and removing the personal protective equipment is critical to the staff safety. ${ }^{27}$ Currently, no specific antiviral treatment is recommended for MERS-CoV infection. ${ }^{26}$ Recently, interferon and ribavirin were introduced in five severe cases with MERS-CoV infection, but they revealed poor outcome due to late initiation of antiviral therapy ${ }^{28}$ Four MERS-CoV infected cases admitted at two South Korean hospitals were injected with blood plasma from recovering patients, but there is insufficient clinical basis about the results of plasma therapy among experts in this country. ${ }^{3}$

\section{Discussion}

MERS characteristically presents as a nonspecific febrile respiratory tract infection. Nevertheless, it can progress rapidly to respiratory failure or it never becomes failure. Comorbidity with pulmonary disease, diabetes, renal disease, and immunodeficiency status are risk factors for disease progression. First reported in Jeddah, KSA in June 2012 and the first outbreak occurred in March or April 2012 in Zarqa, Jordan. ${ }^{29}$ Amending the WHO's International Health Regulations is needed to grant the director general flexibility for designation of graduated alert levels, heightened vigilance, rising to a Public Health Emergency of International Concern only when justified. Transparency give inevitable scientific uncertainty and the health authorities should fully disclose what is and is unknown about the outbreaks of MERS-CoV, since public fear and mistrust in government can impede epidemic response. ${ }^{29}$

\section{Conclusion}

Human-to-human transmission of MERS-CoV occurs via the droplet-contact route. The vast majority of cases are reported from the Middle East, particularly, KSA. Introduction of MERS-CoV infection by a returning traveler result in a large outbreak in the South Korea (182 cases and 32 deaths, as of June 27, 2015). Up to now, there is neither evidence of neither airborne transmission nor sustained human-to-human transmission in the community and no specific antiviral treatment is discovered. 


\section{Acknowledgments}

None.

\section{Conflicts of interest}

None.

\section{References}

1. Middle East Respiratory Syndrome. Center for Health Protection. 2015

2. Public Health England. Risk assessment of Middle-East-RespiratorySyndrome Coronavirus (MERS-CoV). PHE Publications gateway number. 2015;2015341.

3. Kim J. South Korea conducts experimental plasma therapy on MERS patients. Reuters. 2015.

4. Zaki AM, van Boheeman S, Bestebroer TM, et al. Isolation of a novel coronavirus from a man with pneumonia in Saudi Arabia. $N$ Engl J Med. 2012;367(19):1814-1820.

5. United Kingdom Health Protection Agency. Partial genetic sequence information for scientists about the novel coronavirus. 2012

6. Cotton M, Lam TT, Watson SJ, et al. Full-genome deep sequencing and phylogenetic analysis of novel human beta-coronavirus. Emerg Infect Dis. 2013;19(5):736-42B.

7. Annan A, Baldwin HJ, Corman VM, et al. Human beta-coronavirus $2 \mathrm{c}$ EMC/2012-related viruses in bats, Ghana and Europe. Emerg Infect Dis. 2013;19(3):456-459.

8. Ithete NL, Stoffberg S, Corman VM, et al. Close relative of human Middle-East-Respiratory-Syndrome coronavirus in bat, South Africa. Emerg Infect Dis. 2013;19(10):1697-1699.

9. Memish ZA, Mishra N, Olival KJ, et al. Middle-East-RespiratorySyndrome coronavirus in bats, Saudi Arabia. Emerg Infect Dis. 2013;19(11):1819-1823.

10. Chan JF, Lau SK, To KK, et al. Middle-East-Respiratory-Syndrome coronavirus: another zoonotic beta-coronavirus causing SARS-like disease. Clin Microbiol Rev. 2015;28(2):465-522.

11. Raj VS, Mou H, Smits SL, et al. Dipeptidyl peptidase 4 is a functional receptor for the emerging human coronavirus-EMC. Nature. 2013;495(7440):251-254

12. Guangwen L, Yawei H, Qihui W, et al. Molecular basis of binding between novel human coronavirus MERS-CoV and its receptor CD26. Nature. 2013;500:227-231.

13. McIntosh K. Middle-East-Respiratory-Syndrome coronavirus. UpToDate. 2015.

14. Kindler E, Jo'nsdo'ttir HR, Muth D, et al. Efficient replication of the novel human beta-coronavirus EMC on primary human epithelium highlight its zoonotic potential. MBio. 2013;4(1):e00611.

15. Chan JF, Chan KH, Choi GK, et al. Differential cell line susceptibility to the emerging novel human beta-coronavirus 2c EMC/2012 implications for disease pathogenesis and clinical manifestations. $J$ Infect Dis. 2013;207(11):1743-1752.
16. Müller MA, Raj VS, Muth D, et al. Human coronavirus EMC does not require the SARS-coronavirus receptor and maintains broad replicative capability in mammalian cell lines. MBio. 2012;3(6):e000515.

17. Meyer B, Garcia-Bocanegra I, Wernery U, et al. Serologic assessment of possibility for MERS-CoV infection in equids. Emerg Infect Dis. 2015;21(1):181-182.

18. Cotton M, Watson SJ, Kellam P, et al. Transmission and evolution of the Middle-East-Respiratory-Syndrome coronavirus in Saudi Arabia: a descriptive genomic study. Lancet. 2013;382(9909):1993-2002.

19. Ontario Ministry of Health and Long-Term Care. Guidance for Healthcare Workers and Health Sector Employers on Middle-EastRespiratory-Syndrome Coronavirus. 2015

20. Manatu H. Middle-East-Respiratory-Syndrome Coronavirus (MERSCoV). New Zealand Ministry of Health. 2015.

21. Infection prevention/control and management guidelines for patients with Middle-East-Respiratory-Syndrome Coronavirus (MERS-CoV) infection. (2nd edn). Scientific Advisory Council, Ministry of Health, Saudi Arabia. 2014

22. Stephanie RB, Donna A, Ray A, et al. First confirmed case of MiddleEast-Respiratory-Syndrome coronavirus (MERS-CoV) infection in the United States, updated information on epidemiology of MERS$\mathrm{CoV}$ infection, and guidance for the public, clinicians, and public health Authorities-May 2014. MMWR Morb Mortal Wkly Rep. 2014;63(19):431-436

23. World Health Organization. Laboratory testing for Middle-EastRespiratory-Syndrome coronavirus. Interim recommendations (revised). 2014

24. World Health Organization. Interim surveillance recommendations for human infection with Middle-East-Respiratory-Syndrome coronavirus. 2014.

25. United States Centers for Disease Control and Prevention. Interim guidelines for collecting, handling, and testing clinical specimens from patients under investigation (PUIs) for Middle-East-RespiratorySyndrome coronavirus (MERS-CoV)-Version. 2015;2.1.

26. United States Centers for Disease Control and Prevention. Prevention and Treatment of MERS. 2015.

27. Queensland Government, Australia. Interim infection control guidelines for management of Middle-East-Respiratory-Syndrome Coronavirus. 2015.

28. Al-Tawfiq JA, Momattin H, Dib J, et al. Ribavirin and interferon therapy in patients infected with the Middle-East-Respiratory-Syndrome coronavirus: an observational study. Int J Infect Dis. 2014;20:42-46.

29. Lawrence OG, Daniel L. Middle-East-Respiratory-Syndrome: A Global Health Challenge. JAMA. 2015;314(8):771-772. 